\title{
Long-term prognostic utility of low-density lipoprotein (LDL) triglyceride in real-world patients with coronary artery disease and diabetes or prediabetes
}

Jing-Lu Jin ${ }^{1 \dagger}$, Hui-Wen Zhang ${ }^{1 \dagger}$, Ye-Xuan Cao ${ }^{1}$, Hui-Hui Liu' ${ }^{1}$, Qi Hua², Yan-Fang Li ${ }^{3}$, Yan Zhang ${ }^{1}$, Yuan-Lin Guo ${ }^{1}$,

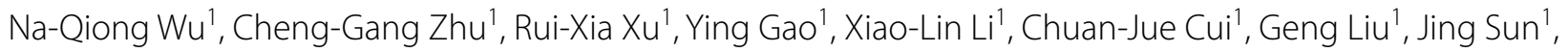
Qian Dong ${ }^{1}$, Raul Santos ${ }^{4,5}$ and Jian-Jun $\mathrm{Li}^{1 *}$ (D)

\begin{abstract}
Background: Recent guidelines highlighted the association between atherosclerosis and triglyceride-enriched lipoproteins in patients with impaired glucose metabolism. However, evidence from prospective studies for long-term prognostic utility of low-density lipoprotein triglyceride (LDL-TG) in real-world patients with prediabetes (Pre-DM) or diabetes mellitus (DM) and coronary artery disease (CAD) is currently not available. The aim of the present study was to evaluate the impact of LDL-TG on major adverse cardiovascular events (MACEs) in patients with stable CAD under different glucose metabolism status.

Methods: A total of 4381 patients with CAD were consecutively enrolled and plasma LDL-TG level was measured by an automated homogeneous assay. They were categorized according to both status of glucose metabolism [DM, Pre-DM, normal glycaemia regulation (NGR)] and tertiles of LDL-TG. All subjects were followed up for the occurrence of MACEs.

Results: During a median of 5.1 (interquartile range 3.9 to 5.9) years' follow-up, 507 (11.6\%) MACEs occurred. Cubic spline models showed a significant association between LDL-TG and MACEs in DM and Pre-DM but not in NGR. When the combined effect of elevated LDL-TG and glucose disorders was considered for risk stratification, the medium tertile of LDL-TG plus DM, and the highest tertile of LDL-TG plus Pre-DM or plus DM subgroups were associated with significantly higher risk of MACEs after adjustment of confounders including triglyceride [hazard ratios (95\% confidence intervals): 1.843 (1.149-2.955), 1.828 (1.165-2.867), 2.212 (1.396-3.507), all $p<0.05]$. Moreover, adding LDL-TG into the original model increased the $C$-statistic from 0.687 to 0.704 ( $\Delta C$-statistic $=0.016, p=0.028$ ) and from 0.734 to $0.749(\Delta C$-statistic $=0.014, p=0.002)$ in Pre-DM and DM, respectively.
\end{abstract}

Conclusions: In this longitudinal cohort study on real-world practice, higher LDL-TG was associated with worse outcomes among Pre-DM and DM patients with stable CAD.

\footnotetext{
*Correspondence: lijianjun938@126.com; 13901010368@163.com

${ }^{\dagger}$ Jing-Lu Jin and Hui-Wen Zhang contributed equally to this work

1 State Key Laboratory of Cardiovascular Disease, Fu Wai Hospital, National

Center for Cardiovascular Diseases, Chinese Academy of Medical Sciences,

Peking Union Medical College, BeiLiShi Road 167, Beijing 100037, China

Full list of author information is available at the end of the article
}

\section{$\triangle B M C$}

(c) The Author(s) 2020. This article is licensed under a Creative Commons Attribution 4.0 International License, which permits use, sharing, adaptation, distribution and reproduction in any medium or format, as long as you give appropriate credit to the original author(s) and the source, provide a link to the Creative Commons licence, and indicate if changes were made. The images or other third party material in this article are included in the article's Creative Commons licence, unless indicated otherwise in a credit line to the material. If material is not included in the article's Creative Commons licence and your intended use is not permitted by statutory regulation or exceeds the permitted use, you will need to obtain permission directly from the copyright holder. To view a copy of this licence, visit http://creativecommons.org/licenses/by/4.0/. The Creative Commons Public Domain Dedication waiver (http://creativecommons.org/publicdomain/zero/1.0/) applies to the data made available in this article, unless otherwise stated in a credit line to the data. 
Keywords: LDL-TG, Stable CAD, Diabetes, Pre-diabetes, MACEs

\section{Background}

Genetic studies have verified that both plasma triglyceride (TG) levels or genetic variants leading to hypertriglyceridemia are independently associated with higher risk of coronary artery disease (CAD) [1, 2]. In the statin era, even among those with low density lipoprotein cholesterol (LDL-C) within recommended values, elevation in plasma TG still indicates an elevated residual risk. Hence, TG-enriched lipoproteins could be a causal risk factor for cardiovascular events [3]. However, in randomized clinical trials, inconsistent results about CAD risk reduction were reported among patients receiving TG lowering therapies [4-6]. Although TGs might not be atherogenic per se, several studies showed that TG-enriched lipoproteins were strongly related with the occurrence of atherosclerotic cardiovascular disease (ASCVD) events [7-9].

Low density lipoprotein triglyceride (LDL-TG), which represents the TG content of LDL particles, was previously reported to be associated with incident cardiovascular disease. In the Atherosclerosis Risk in Communities (ARIC) study, the prognostic value of LDL-TG trumped that of remnant cholesterol (RC) in patients without CAD at baseline [7]. As it was reported by McKeone et al., LDL with elevated TG contents formed in vitro had reduced proteolytic cleavage of apo B-100 [10]. For realworld individuals, plasma levels of LDL-TG were elevated when more TGs were transferred from very low density lipoprotein (VLDL) to LDL via cholesteryl ester transfer protein (CETP) [11]. Notably, this hypertriglyceridemiamediated pathway was more active when individuals presented glycemic metabolism disorders [11, 12].

Diabetes mellitus (DM) is a common disease which confers a two-fold excess risk of vascular diseases after adjustment for confounders [13]. In type 2 diabetes mellitus(T2DM) patients, the macrovascular complications are due in part to hyperglycemia, but the coexistence of other disorders may result in worse prognosis [14, 15]. The morbidity rate of pre-diabetes (Pre-DM), a state of mild glucose dysregulation, is also increasing worldwide. The incidence rate of Pre-DM is almost three-fold higher than that of DM [16]. Interestingly, although PreDM individuals present modestly raised glucose levels, similar cardiac abnormalities of DM subjects were also observed in these patients. For example, Coopmans et al. has reported that Pre-DM and DM are similarly associated with reduction of heart rate variability [17]. Previous studies including ours have also indicated that patients with DM or Pre-DM have similar pro-atherogenic lipid profiles [12]. Compared with normal glucose regulation
(NGR) individuals, lipid abnormalities, especially for hypertriglyceridemia and elevated lipoprotein (a), were more significantly associated with cardiovascular events in DM or Pre-DM patients $[14,18]$. Moreover, several studies indicated that increased plasma LDL-TG level was one of the typical changes encountered in patients with impaired glucose metabolism [12]. This raised the possibility that higher LDL-TG levels may have different impacts on the prognosis of patients with NGR, Pre-DM and DM. In the current study, we aim to investigate the different relations of LDL-TG levels to major cardiovascular events (MACEs) in stable CAD patients with different glucose metabolism status under real-world clinical practice.

\section{Method \\ Study design and participants}

This study complied with the Declaration of Helsinki and was approved by the local ethical review board. Informed written consents were obtained from all participants.

Study patient disposition is described in the flowchart at Additional file 1: Figure S1. From March 2011 to December 2016, 5132 patients were admitted to three medical centers and diagnosed as angiographyproven CAD. Seven-hundred and fifty-one patients did not enter the final analysis for the following exclusion criteria: missing data, having suffered an acute coronary syndrome (ACS) or previous revascularization, decompensated heart failure, severe liver and/or renal insufficiency, thyroid dysfunction, systematic inflammatory disease, malignant disease, and chylomicronemia. Patients were followed up by telephone or presential interviews at 6 months' intervals by experienced nurses or physicians. Medical records of those who reported MACEs were checked by experienced physicians who were blinded to the current study. The MACEs were defined as cardiovascular mortality, non-fatal myocardial infarction (MI), atherothrombotic stroke, unplanned percutaneous coronary intervention (PCI) or coronary artery bypass grafting (CABG), and hospitalization for unstable angina. Non-fatal myocardial infarction was diagnosed as positive cardiac enzymes along with typical chest pain or electrocardiogram serial changes. Stroke was diagnosed by medical history, typical symptoms, and characterized imaging.

DM was diagnosed by fasting plasma glucose (FPG) $\geq 7.0 \mathrm{mmol} / \mathrm{L}$ or the 2 -h plasma glucose of the oral glucose tolerance test $\geq 11.1 \mathrm{mmol} / \mathrm{L}$, haemoglobin A1c (HbA1c) level $\geq 6.5 \%$ or currently using anti-diabetic 
drugs or insulin. Pre-DM was diagnosed in participants who did not have medical history of DM but met the American Diabetes Association(ADA) criteria of Pre-DM [fasting plasma glucose 5.6 to $6.9 \mathrm{mmol} / \mathrm{L}, 2$-h glucose ranging from 7.8 to $11.0 \mathrm{mmol} / \mathrm{L}$, or hemoglobin A1c (HbA1c) level from 5.7 to 6.4\%] [19]. Patients without DM or pre-DM were categorized as NGR. Hypertension was defined as medical history of hypertension, currently receiving antihypertensive drugs or hospital recorded systolic blood pressure (SBP) $\geq 140 \mathrm{mmHg}$ and/or diastolic blood pressure (DBP) $\geq 90 \mathrm{mmHg}$ for three or more consecutive times. Other clinical characters, including family history of early cardiovascular disease, prior medications, smoking, and alcohol consumption, were collected from self-reported or hospital-recorded medical history.

\section{Laboratory analysis}

Blood samples were obtained after 12-h fasting once upon admission and were collected into EDTA-containing tubes. After centrifugation at $3000 \mathrm{rpm}$ for $10 \mathrm{~min}$ at $4{ }^{\circ} \mathrm{C}$, plasma was collected and stored at $-80^{\circ} \mathrm{C}$. Plasma concentrations of total cholesterol (TC), TG, LDL-C, high density lipoprotein cholesterol (HDL-C), apolipoprotein $B$ (ApoB) were measured by automatic biochemistry analyzer (Hitachi 7150, Tokyo, Japan) in an enzymatic assay. Non-HDL-C was calculated as TC minus HDL-C. Plasma levels of LDL-TG were measured by an automated homogeneous assay (DENKA SEIKEN CO., LTD, Tokyo, Japan) [20]. The measured LDL-TG was with calibration range of $0.0-80 \mathrm{mg} / \mathrm{dL}$. The automated homogeneous LDLTG method used for our study was validated against the standard sequential density ultracentrifugation method [20]. The concentrations of glucose were measured by enzymatic hexokinase method. HbA1c was measured using Tosoh Automated Glycohemoglobin Analyser (HLC-723G8, Tokyo, Japan).

\section{Evaluation of CAD severity}

Angiographic data were collected according to catheterization laboratory records. Three experienced interventional physicians evaluated the result of coronary angiography for each patient, and CAD severity was determined using the Gensini score (GS) as previously described [21].

\section{Statistical analysis}

The values for the continuous variables and the categorical variables were presented as the mean \pm standard deviation (SD), median (Q1-Q3) or number (percentage). The Kolmogorov-Smirnov test was used to test the distribution pattern. The differences of variables among groups were analyzed using Student $t$ test, analysis of variance, or nonparametric test where appropriate. The Kaplan-Meier method was used to estimate the event-free survival rates among. The logrank test was used to test the statistical significance. The hazard ratios (HRs) and 95\% confidence intervals (CI) were calculated by univariate and multivariate Cox regression analyses. The association of LDL-TG concentration with MACEs occurrence was tested either as categorical (divided in low (T1), medium (T2) and high (T3) to tertiles) or continuous (per 1-SD) variables. Both Kaplan-Meier method and Cox regression analyses were performed in subgroups according to DM status and tertiles of LDL-TG levels. HRs for MACEs were also calculated for participants who were divided into 9 groups by both status of glucose metabolism and LDLTG levels using NGR plus low LDL-TG as reference. The associations between LDL-TG concentrations and MACEs of different glucose metabolism groups were also assessed by restricted cubic spline models. The concordance index $(C$-statistic $)$ was calculated to test the model efficiency. The Cox analyses were also performed in DM patients to compare the HRs between those with different glucose control status and LDLTG levels with good glucose control (HbA1c $<7 \%)$ plus low LDL-TG subgroup. The adjustments in multivariate models included age, sex, BMI, smoking, hypertension, family history of early CAD, Gensini score, LVEF, LDL-C HDL-C, TG and baseline use of statins. A p-value $<0.05$ was considered statistically significant. The statistical analyses were performed with SPSS version 21.0 software (SPSS Inc., Chicago, IL, USA) and R language version 3.5.2 (Feather Spray).

\section{Results}

\section{Baseline characteristics}

Patients were divided into three groups according to different glucose metabolism status (NGR: $n=926$, Pre-DM: $n=1789$, DM $n=1666$, Table 1). Older and female patients were more likely to have Pre-DM and $\mathrm{DM}(\mathrm{p}<0.001$ and $\mathrm{p}=0.003$, respectively). The plasma levels of glucose, HbA1c, TG, LDL-TG and proportion of hypertension increased while the levels of HDL-C decreased according to different glucose metabolism status (all $\mathrm{p}<0.001$ ). Patients with Pre-DM had higher TC, LDL-C and non-HDL-C than those with NGR $(\mathrm{p}<0.05)$. DM but not Pre-DM group showed higher body mass index (BMI) and GS but lower left ventricle ejection fraction (LVEF) and lower rate of early family history of CAD than NGR group. Non-significant differences were found regarding ApoB levels, alcohol consumption, smoking and use of medications among NGR, Pre-DM and DM subgroups $(\mathrm{p}>0.05)$. 
Table 1 Baseline clinical and laboratory characteristics according to glucose metabolism status

\begin{tabular}{|c|c|c|c|c|c|}
\hline Variables & Total $n=4381$ & NGR $n=926$ & Pre-DM $n=1789$ & $\mathrm{DM} n=1666$ & $p$ value \\
\hline \multicolumn{6}{|l|}{ Clinical characteristics } \\
\hline Age, years & $58.2 \pm 9.8$ & $55.5 \pm 9.9$ & $58.5 \pm 9.5$ & $59.3 \pm 9.8$ & $<0.001$ \\
\hline Male sex, n (\%) & $3120(71.2)$ & $700(75.6)$ & $1263(70.6)$ & $1157(69.4)$ & 0.003 \\
\hline $\mathrm{BMI}\left(\mathrm{kg} / \mathrm{m}^{2}\right)$ & $25.9 \pm 3.1$ & $25.5 \pm 3.1$ & $25.7 \pm 3.1$ & $26.4 \pm 3.1$ & $<0.001$ \\
\hline Hypertension, n (\%) & $2857(65.2)$ & $536(57.9)$ & $1141(63.8)$ & $1180(70.8)$ & $<0.001$ \\
\hline Family history of early CAD, n (\%) & $610(13.9)$ & $150(16.2)$ & $246(13.8)$ & $214(12.8)$ & 0.059 \\
\hline Current smoker, n (\%) & $2373(54.2)$ & $511(55.2)$ & $962(53.8)$ & $900(54.0)$ & 0.774 \\
\hline Alcohol consumption, n (\%) & $1400(32.0)$ & $311(33.5)$ & $576(32.2)$ & $513(30.8)$ & 0.330 \\
\hline \multicolumn{6}{|l|}{ Laboratory findings } \\
\hline Glucose (mmol/L) & $6.2 \pm 2.0$ & $4.9 \pm 0.6$ & $5.3 \pm 0.7$ & $7.8 \pm 2.4$ & $<0.001$ \\
\hline $\mathrm{HbA} 1 \mathrm{c}(\%)$ & $6.5 \pm 1.2$ & $5.4 \pm 0.2$ & $6.0 \pm 0.2$ & $7.7 \pm 1.2$ & $<0.001$ \\
\hline Creatinine $(\mu \mathrm{mol} / \mathrm{L})$ & $77.6 \pm 15.7$ & $78.3 \pm 15.4$ & $77.1 \pm 15.1$ & $77.8 \pm 16.6$ & 0.149 \\
\hline $\mathrm{TC}(\mathrm{mg} / \mathrm{dL})$ & $159.88 \pm 41.62$ & $157.93 \pm 41.23$ & $161.82 \pm 42.79$ & $158.71 \pm 41.23$ & 0.040 \\
\hline $\mathrm{HDL}-\mathrm{C}(\mathrm{mg} / \mathrm{dL})$ & $40.85 \pm 11.28$ & $42.01 \pm 12.45$ & $43.71 \pm 11.28$ & $39.68 \pm 10.50$ & $<0.001$ \\
\hline LDL-C (mg/dL) & $97.25 \pm 36.57$ & $95.31 \pm 36.18$ & $98.81 \pm 37.34$ & $96.47 \pm 35.40$ & 0.036 \\
\hline Non-HDL-C (mg/dL) & $119.03 \pm 40.07$ & $115.92 \pm 40.07$ & $120.20 \pm 40.56$ & $119.03 \pm 39.68$ & 0.020 \\
\hline ApoB (g/L) & $0.88(0.71-1.08)$ & $0.86(0.70-1.07)$ & $0.88(0.71-1.09)$ & $0.88(0.72-1.08)$ & 0.405 \\
\hline $\mathrm{TG}(\mathrm{mmol} / \mathrm{L})$ & $1.49(1.10-2.11)$ & $1.39(1.02-1.98)$ & $1.52(1.12-2.10)$ & $1.53(1.15-2.19)$ & $<0.001$ \\
\hline LDL-TG (mg/dL) & $17.93 \pm 6.53$ & $16.77 \pm 6.69$ & $17.83 \pm 6.45$ & $18.67 \pm 6.44$ & $<0.001$ \\
\hline $\operatorname{LVEF}(\%)$ & $64.4 \pm 7.2$ & $65.0 \pm 6.5$ & $64.9 \pm 6.8$ & $63.5 \pm 7.9$ & $<0.001$ \\
\hline GS & $22(10-38)$ & $20(11-34)$ & $20(10-36)$ & $24(10-42)$ & 0.002 \\
\hline \multicolumn{6}{|l|}{ Medications } \\
\hline Statins, n (\%) & $3237(73.9)$ & $675(72.9)$ & $1310(73.2)$ & $1252(75.1)$ & 0.324 \\
\hline Statins at follow-up, n (\%) & $4250(97.0)$ & $892(96.3)$ & $1744(97.5)$ & $1614(96.9)$ & 0.226 \\
\hline Aspirin, n (\%) & $2684(61.3)$ & $554(59.8)$ & $1083(60.5)$ & $1037(62.2)$ & 0.411 \\
\hline ACEIs/ARBs, n (\%) & $1266(28.9)$ & $261(28.2)$ & $517(28.9)$ & $488(29.3)$ & 0.838 \\
\hline$\beta$-blockers, n (\%) & $2394(54.6)$ & $495(53.5)$ & $961(53.7)$ & $938(56.3)$ & 0.224 \\
\hline \multicolumn{6}{|l|}{ Antidiabetes drugs } \\
\hline OADs, n (\%) & $1020(23.3)$ & - & - & $1020(61.2)$ & - \\
\hline Insulin, n (\%) & $561(12.8)$ & - & - & $561(33.7)$ & - \\
\hline
\end{tabular}

Data were expressed as mean \pm SD, median with 25th and 75th percentiles or $n$ (\%). ACEls, ACE inhibitors; ARBs, angiotensin receptor blockers; OADs, oral antidiabetes drugs

\section{Glucose metabolism status, lipid parameters and coronary severity}

The coronary severity was assessed by GS in different subgroups according to glucose metabolism status and LDL-TG levels. As shown in Additional file 1: Figure S2A, DM group had significantly higher GS than NGR group $(\mathrm{p}<0.001)$. However, no significant difference in GS was observed between pre-DM and NGR groups $(\mathrm{P}>0.05)$. In the meanwhile, high (T3) but not medium (T2) LDL-TG subgroups had higher GS than low LDL-TG subgroup (T1, Additional file 1: Figure S2B).

In both univariate and multivariate linear regression analyses (Additional file 1: Table S1), the HDL-C levels were negatively correlated with GS $(\mathrm{p}<0.001$, respectively). However, no relationship between plasma TG levels and coronary severity was found. Other lipid parameters, including TG, LDL-C, HDL-C, non-HDL-C,
ApoB, and LDL-TG were positively correlated with the severity of CAD (all $\mathrm{p}<0.05)$.

\section{Predictive value of LDL-TG and other lipid parameters for cardiovascular outcomes}

Overall, during a median follow-up of 5.1 (interquartile range 3.9 to 5.9) years, 507 MACEs occurred, including 75 cardiovascular deaths (14.8\%), 52 nonfatal MIs (10.3\%), 110 nonfatal strokes (21.7\%), 168 unplanned revascularizations (33.1\%) and 102 (20.1\%) unstable angina hospitalizations. The incidence rate of MACEs was 25.1 (95\% CI 23.2-27.3) per 1000 person years.

As shown in Additional file 1: Table S2, for the total population, 1-SD increment of LDL-TG was associated with $26.8 \%$ and $32.8 \%$ higher risk of outcomes in univariate and multivariate models respectively (HR per 1-SD increment: 1.268 , 95\% CI 1.174-1.370 and adjusted HR 
per 1-SD increment: $1.328,95 \%$ CI 1.202-1.467). Compared with other lipid parameters including TG, LDL-C, HDL-C, non-HDL-C, and ApoB, the prognostic value of LDL-TG was more significant (Table 2).

\section{LDL-TG, different glucose metabolism status and occurrence of MACEs}

Kaplan-Meier analysis (Fig. 1a) showed that DM subjects had significantly lower event-free survival rates than NGR $(P<0.05)$, while there were non-significant differences between those of pre-DM and NGR. Both medium (T2) and high LDL-TG subgroups (T3) had higher event rates than low LDL-TG subgroup (T1, Fig. 1b).

Restricted cubic spline models showed that no association between LDL-TG and MACEs existed in NGR group (Fig. 2a). On the other hand, pre-DM and DM groups presented increasing associations between LDL-TG and MACEs while such association was stronger in the DM group (Fig. 2b, c). As presented in Fig. 3, LDL-TG (per SD increment) as a continuous variable was positively associated with MACEs occurrence in patients with Pre-DM and DM (adjusted HR 1.391, 95\% CI 1.170-1.654 and HR: 1.597 , 95\% CI 1.347-1.893, respectively, all $\mathrm{p}<0.05$ ) but not in those with NGR (HR 1.074, 95\% CI $0.846-$ $1.364 \mathrm{p}>0.05)$. When LDL-TG was analyzed according to tertiles (low, medium and high LDL-TG for T1 to T3), high LDL-TG subgroups presented 1.559-fold and 2.078-fold higher risk of MACEs than low LDL-TG subgroups in patients with Pre-DM and DM. Medium LDLTG (T2) had higher risk of MACEs in DM (adjusted HR: $1.645,95 \%$ CI 1.120-2.417, $\mathrm{p}<0.05$ ) but not in Pre-DM (adjusted HR: 1.153, 95\% CI 0.786-1.691, p >0.05). Both high and medium LDL-TG subgroups did not present higher events risk in NGR (all p > 0.05).

As presented in Additional file 1: Table S3, univariate Cox regression models showed that patients with DM had 1.529-fold higher risk of MACEs while pre-DM group did not present increment in MACEs. Additional

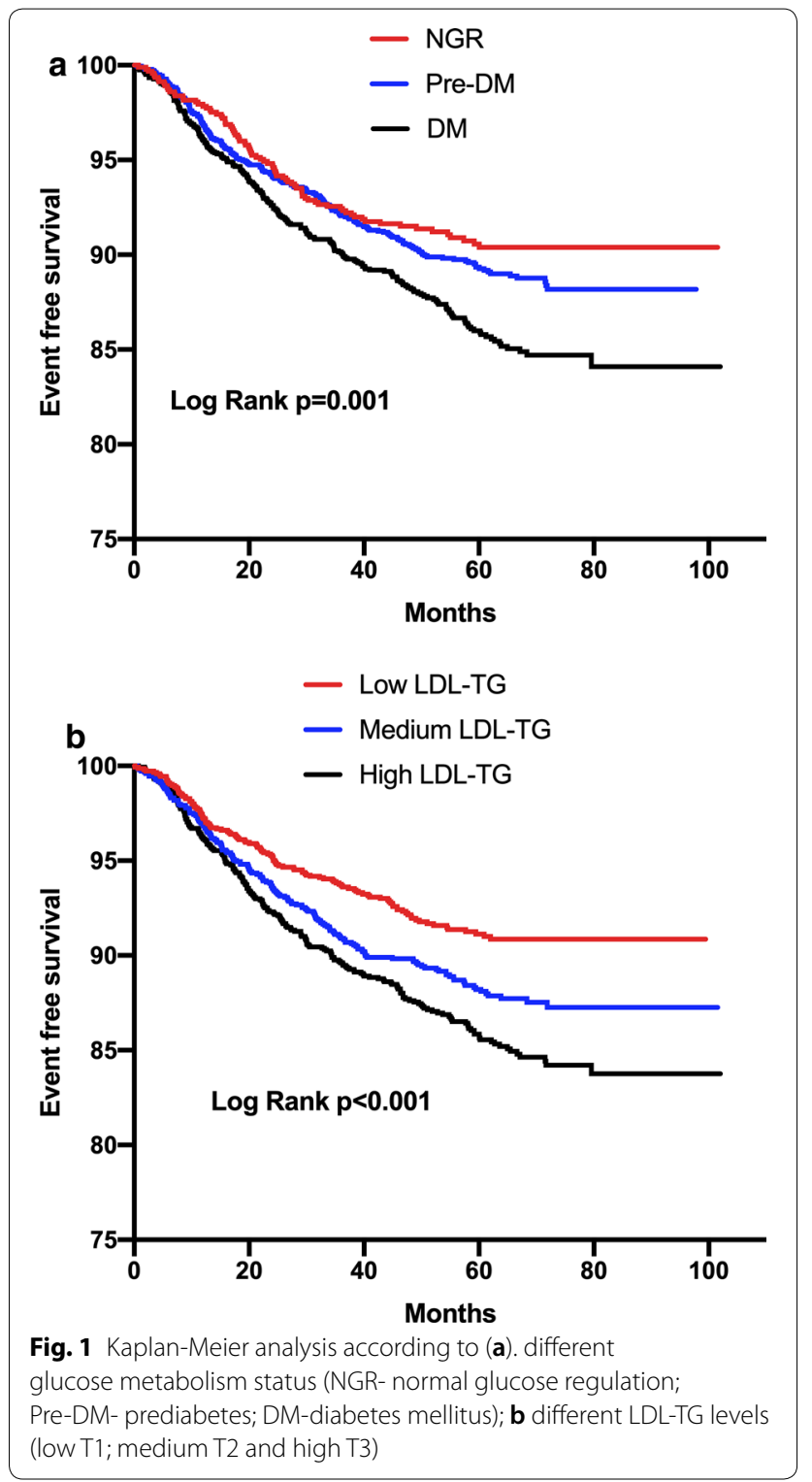

Table 2 Univariate and multivariate Cox proportional hazards regression analysis of the lipid parameters with MACEs

\begin{tabular}{|c|c|c|c|c|c|}
\hline \multirow[t]{2}{*}{ Variables } & \multirow[t]{2}{*}{ Per-SD } & \multicolumn{2}{|c|}{ Univariate Cox regression } & \multicolumn{2}{|c|}{ Multivariate Cox regression } \\
\hline & & $\mathrm{HR}(95 \% \mathrm{Cl})$ & $\mathbf{P}$ & $\mathrm{HR}(95 \% \mathrm{Cl})$ & $\mathbf{P}$ \\
\hline TG & $1.02 \mathrm{mg} / \mathrm{dL}$ & $1.087(1.002-1.180)$ & 0.046 & $1.020(0.930-1.119)$ & 0.676 \\
\hline LDL-C & $36.57 \mathrm{mg} / \mathrm{dL}$ & $1.116(1.027-1.212)$ & 0.009 & $1.091(1.002-1.189)$ & 0.046 \\
\hline $\mathrm{HDL}-\mathrm{C}$ & $40.85 \mathrm{mg} / \mathrm{dL}$ & $0.976(0.893-1.067)$ & 0.599 & $0.952(0.865-1.048)$ & 0.316 \\
\hline Non-HDL-C & $40.07 \mathrm{mg} / \mathrm{dL}$ & $1.108(1.020-1.204)$ & 0.016 & $1.096(1.005-1.195)$ & 0.038 \\
\hline ApoB & $0.29 \mathrm{~g} / \mathrm{L}$ & $1.089(1.006-1.179)$ & 0.034 & $1.069(0.987-1.159)$ & 0.102 \\
\hline LDL-TG & $6.53 \mathrm{mg} / \mathrm{dL}$ & $1.268(1.174-1.370)$ & $<0.001$ & $1.310(1.192-1.439)$ & $<0.001$ \\
\hline
\end{tabular}

Italics values indicate statistically significant

Multivariate adjustments include age, sex, body mass index, smoking, hypertension, diabetes mellitus, family history of early coronary artery disease, Gensini score, left ventricular ejection fraction, and baseline statin use 

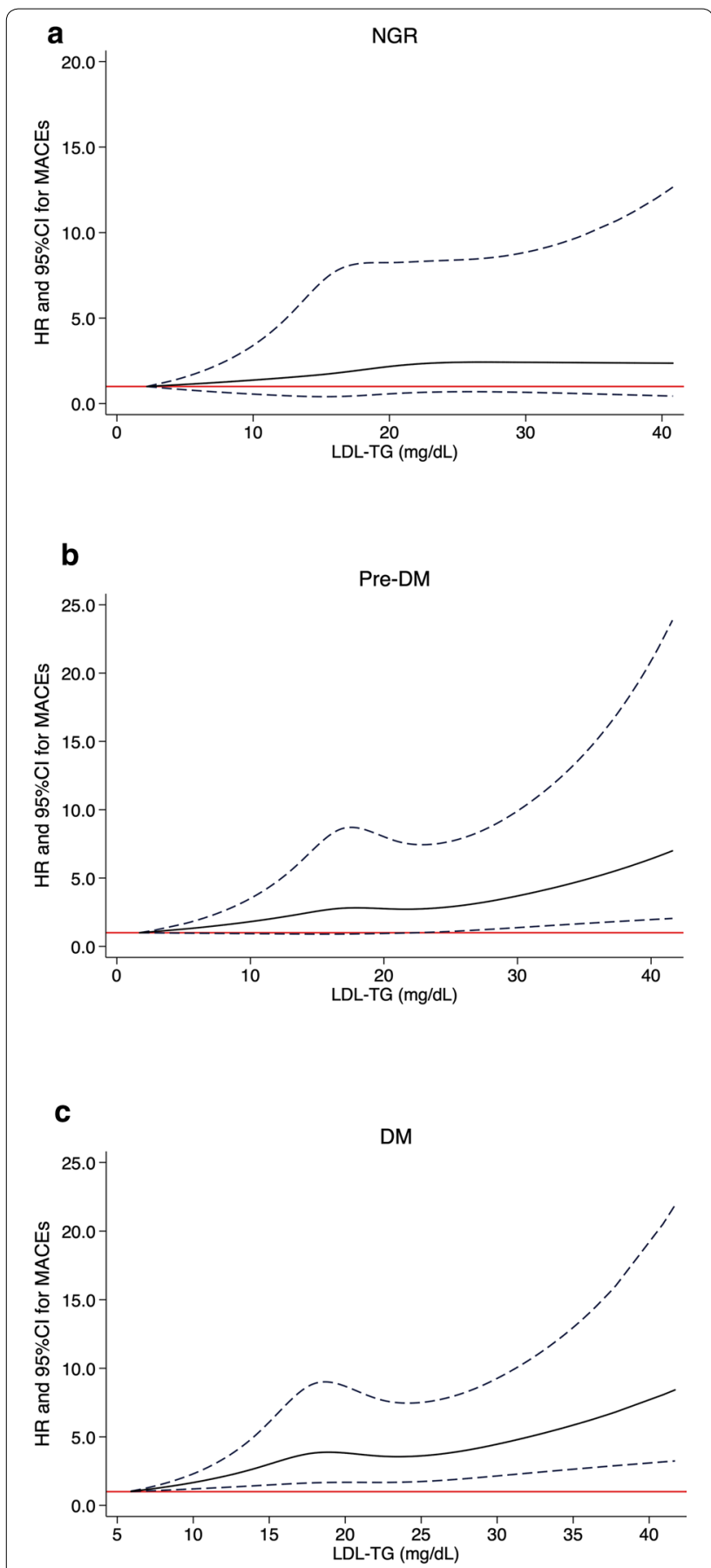

Fig. 2 Restricted cubic spline plot of the association between LDL-TG levels and the risk of MACEs in $\mathbf{a}$ NGR $\mathbf{b}$ Pre-DM and $\mathbf{c}$ DM groups. Solid lines: hazard ratio (HR); dashed lines: 95\% confidence limits (CL); red lines: reference

adjustment for age and sex or traditional confounders did not change the association. However, when both glucose metabolism and LDL-TG status were incorporated as stratifying factors, multivariate adjusted Cox regression analyses indicated that patients in DM plus medium (T2) LDL-TG, pre-DM plus high LDL-TG (T3) and DM plus high LDL-TG (T3) had higher risk of MACEs than individuals with NGR plus low LDL-TG respectively [HR(95\% CI) 1.843 (1.149-2.955), 1.828 (1.165-2.867), $2.212(1.396-3.507)$, all $\mathrm{p}<0.05$, Table 3].

Including LDL-TG to the risk model of traditional CAD risk factors improved the model efficiency by $0.017[\Delta \mathrm{C}$-statistic and $95 \% \mathrm{CI} 0.016(0.004-0.033)$, C-statistic and 95\% CI 0.704 (0.664-0.742), $\mathrm{p}=0.028$, Additional file 1: Table S4] and 0.014[ $\Delta \mathrm{C}$-statistic and $95 \% \mathrm{CI}$ 0.014 (0.006-0.024), C-statistic and 95\% CI 0.749 (0.715$0.783), p=0.002]$ in Pre-DM and DM, respectively.

\section{LDL-TG and MACEs occurrence according to DM control status}

In the subgroup of controlled DM (defined as HbA1c $<7 \%$ $\mathrm{n}=572,34.3 \%$ ), both medium (T2) and high LDL-TG (T3) had no association with MACEs after adjustment for other risk factors. Meanwhile, among patients with uncontrolled DM ( $\mathrm{n}=1094,65.7 \%)$, high LDL-TG (T3) but not medium LDL-TG (T2) was associated with higher risk of MACEs occurrence in fully adjusted models (High LDL-TG: adjusted HR: 1.932, 95\% CI 1.150-3.245, $\mathrm{p}<0.05$, Fig. 4a). When DM patients were categorized into 6 groups according to both levels of HbA1c (7\% as cut-off) and LDL-TG levels (Fig. 4b, c), those in controlled DM plus high LDL-TG, uncontrolled DM plus medium LDL-TG, and uncontrolled DM plus high LDLTG had 2.082-fold (95\% CI 1.055-4.106, p < 0.05), 1.874fold (95\% CI 1.077-3.451, $\mathrm{p}<0.05)$ and 2.171 -fold (95\% CI 1.152-4.090, $\mathrm{p}<0.05$ ) higher risk of MACEs in multivariate adjusted models.

\section{Discussion}

Previous study reported that plasma LDL-TG level was predictive of incident CAD in community-based populations [7]. In patient with DM, the malignant nature of diabetic dyslipidemia may be easily revealed by TGs in lipoprotein classes. In this real-world study, it was demonstrated for the first time that LDL-TG could independently predict the occurrence of MACEs in stable CAD patients with Pre-DM and DM but not in those with NGR. The results persisted after adjustment for robust risk markers including TG,LDL-C, HDL-C, coronary severity and use of lipid lowering therapies.

Hypertriglyceridemia is a common form of dyslipidemia worldwide. Approximately $30 \%$ of adult population in Western societies have elevated TG $(>1.7 \mathrm{mmol} / \mathrm{L})$ [22]. In China, the prevalence of hypertriglyceridemia also increased from 5.7\% to $15 \%$ from 2002 to 2015 [23]. Although the association between TG and CAD risk was supported by solid evidence from genome-wide analyses 


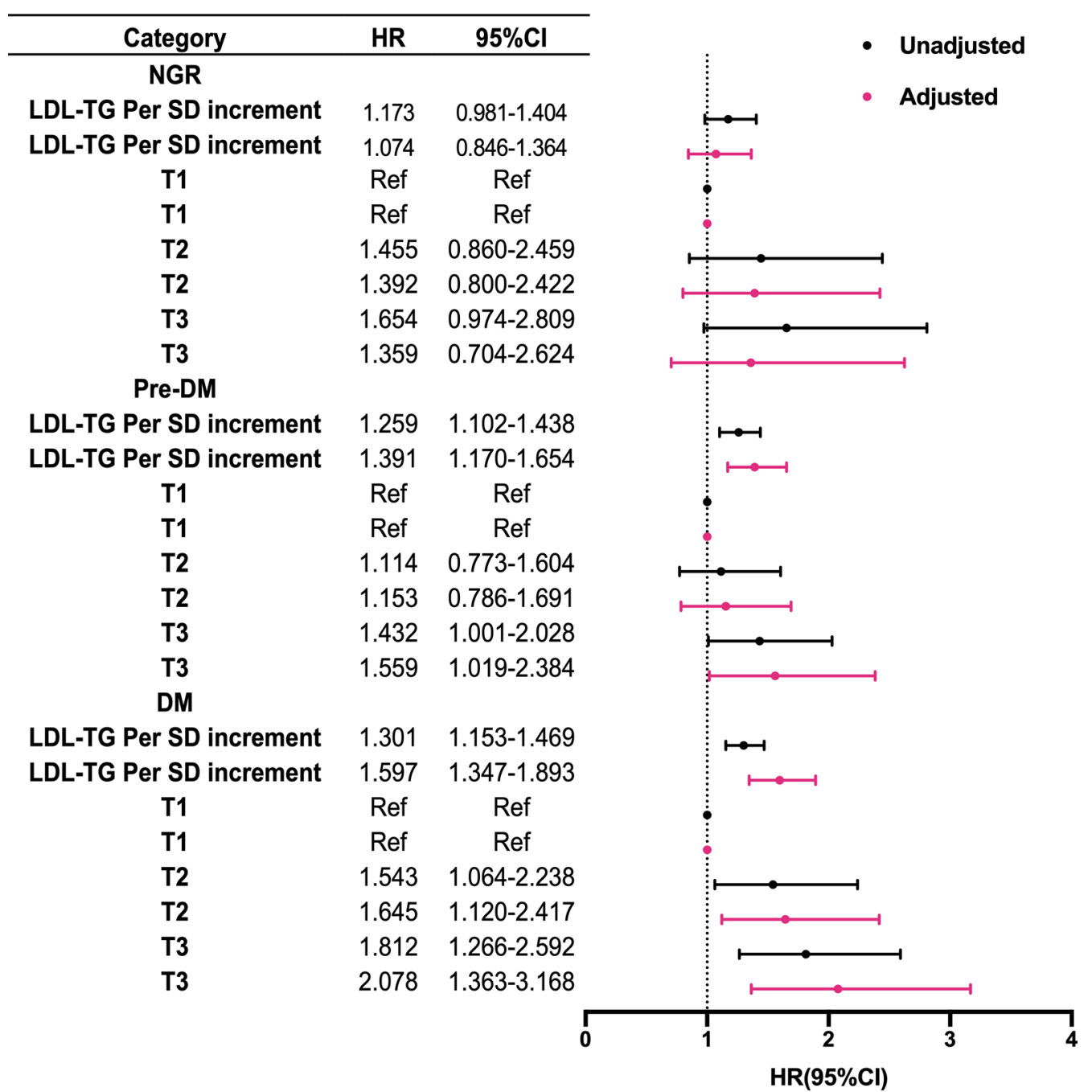

Fig. 3 Predictive value of continuous and categorical LDL-TG in different glucose metabolism status. Glucose metabolism status (NGR- normal glucose regulation; Pre-DM- pre diabetes; DM-diabetes mellitus); LDL-TG levels (low T1; medium T2 and high T3); adjusted variables include age, sex, body mass index, smoking, hypertension, family history of early coronary artery disease, Gensini score, left ventricular ejection fraction, LDL-cholesterol, HDL-cholesterol, triglyceride and baseline statins

and Mendelian randomization studies, several TG lowering therapies had failed to provide cardiovascular benefits in randomized clinical trials. For example, $1 \mathrm{~g}$ of $\mathrm{n}-3$ fatty acids daily did not associate with conclusive effect on cardiovascular risk reduction in Outcome Reduction with an Initial Glargine Intervention (ORIGIN) trial [5]. However, in Reduction of Cardiovascular Events with Icosapent Ethyl-Intervention Trial (REDUCE-IT), patients who received $2 \mathrm{~g}$ of icosapent ethyl twice daily had $25 \%$ lower risk of ischemic events than placebo group [6]. Recently, genetic analysis indicated that the clinical benefit of lipid lowering therapy might attribute to the absolute change of ApoB-containing particles, which was mostly referred to cholesterol-rich LDL or TG-rich VLDL [24]. Thus, TG lowering drugs which could achieve specific reductions in TG-rich VLDL and its related parameters, might be beneficial [25]. In fact, in hypertriglyceridemic status, by activating CETP, the TGs from VLDL were transferred to LDL in exchange for cholesteryl esters, which led to increment in plasma LDL-TG levels [11]. Hence, finding the predictive value of LDL-TG in cardiovascular outcomes may provide new insight into the significance of TG lowering therapy.

In previous studies, controversial results were reported about the independent role of all TGs for predicting cardiovascular outcome. In Copenhagen Ischemic Heart Disease Study, increased concentrations of both calculated and measured RC 
Table 3 LDL-TG levels in relation to cardiovascular events in patients with different glucose metabolism status

\begin{tabular}{|c|c|c|c|}
\hline \multirow[t]{2}{*}{ LDL-TG } & \multirow{2}{*}{$\begin{array}{l}\text { Events/subjects } \\
507 / 4381\end{array}$} & \multicolumn{2}{|l|}{$\mathrm{HR}(95 \% \mathrm{Cl})$} \\
\hline & & Crude model & Adjusted model \\
\hline \multicolumn{4}{|l|}{ NGR } \\
\hline Low LDL-TG (T1) & $26 / 378$ & Ref & Ref \\
\hline Medium LDL-TG (T2) & $30 / 296$ & $1.443(0.853-2.440)$ & $1.491(0.877-2.535)$ \\
\hline High LDL-TG (T3) & $29 / 252$ & $1.654(0.974-2.808)$ & $1.663(0.958-2.866)$ \\
\hline \multicolumn{4}{|l|}{ Pre-DM } \\
\hline Low LDL-TG (T1) & $54 / 603$ & $1.278(0.800-2.040)$ & $1.155(0.723-1.846)$ \\
\hline Medium LDL-TG (T2) & $62 / 608$ & $1.443(0.913-2.282)$ & 1.368 (0.859-2.179) \\
\hline High LDL-TG (T3) & $77 / 578$ & $1.873(1.201-2.923)^{*}$ & $1.843(1.149-2.955)^{*}$ \\
\hline \multicolumn{4}{|l|}{$\mathrm{DM}$} \\
\hline Low LDL-TG (T1) & $42 / 460$ & $1.343(0.824-2.191)$ & $1.116(0.682-1.825)$ \\
\hline Medium LDL-TG (T2) & $82 / 581$ & $2.084(1.341-3.240)^{*}$ & $1.828(1.165-2.867)^{*}$ \\
\hline High LDL-TG (T3) & $105 / 625$ & $2.457(1.600-3.775)^{*}$ & $2.212(1.396-3.507)^{*}$ \\
\hline
\end{tabular}

Model adjusted for age, sex, body mass index, smoking, hypertension, diabetes mellitus, family history of early coronary artery disease, Gensini score, left ventricular ejection fraction, LDL-cholesterol, HDL-cholesterol, triglycerides and baseline use of statins; ${ }^{*}$ for $\mathrm{p}<0.05$

were associated with increased all-cause mortality in patients with ischemic heart disease. However, according to Saeed et al. in ARIC study, RC and LDL-TG were associated with cardiovascular risk, but this association vanished for RC adjusted traditional risk factors including lipids. In a recent prospective study by Silbernagel et al. using the LURIC cohort (3140 participants and mean follow-up time 8.8 years), the fourth and fifth quantiles of LDL-TG had increased risk of cardiovascular mortality while no such association existed for the highest two quantiles of TG and VLDL-TG [26]. These studies indicated that LDL-TG may be the most powerful marker in predicting cardiovascular risk among all the TGs in lipoprotein subclasses. On the contrary, in the Atherothrombosis Intervention in Metabolic Syndrome with Low HDL/High Triglycerides and Impact on Global Health Outcomes (AIM-HIGH) trial, which examined the relationship of LDL-TG and cardiovascular events in a dyslipidemic population, LDL-TG was not associated with worse cardiovascular outcome [27]. However, on the same token,traditional lipid parameters, including LDL-C, HDL-C and non-HDL$\mathrm{C}$, were not predictive of clinical cardiovascular events either [28]. One of the possible reasons for these negative results was that niacin-induced compositional changes in lipoproteins might have affected the atherogenicity of the LDL-TG. Our real-world study, which was conducted in a large cohort of Chinese patients with stable CAD, not only validated the results from ARIC and LURIC studies but also demonstrated that the predictive value of LDL-TG for MACEs was superior to traditional lipid parameters including TG, LDLC, non-HDL-C and ApoB.
The morbidity rate of DM and Pre-DM is increasing rapidly worldwide. The number of individuals with DM and Pre-DM may both reach 600 million in 2045 [13]. The coexistence of DM or Pre-DM and CAD is common. According to recent guidelines, patients with both ASCVD and DM are considered to be at a very high risk of cardiovascular events recurrence and mortality. Intensive LDL-C lowering treatments brought favorable cardiovascular outcome in those patients [29-31]. For example, achieving LDL-C $<1.4 \mathrm{mmol} / \mathrm{L}$ for those with combined status of DM and ASCVD was highly recommended by recent European guidelines on diabetes, prediabetes, and cardiovascular diseases [13]. However, even after intensive lowering of LDL-C, the absolute risk of events in DM patients is still higher than that in non-diabetic patients. Hypertriglyceridemia is the main feature of lipid disorder in DM and Pre-DM and could induce a sequence of compositional changes for lipid parameters including low HDL-cholesterol and type B pattern of LDL distribution [32, 33]. Despite the undisputable causal role of elevated LDL-C in patients with DM [34], TG-enriched lipoproteins were significantly elevated while plasma levels of LDL-C were usually within the normal range [12]. Considering the increased cardiovascular event rate in DM and pre-DM patients, identifying whether compositional features of diabetic dyslipidemia were impedimental factors for further risk reduction was crucial. Until now, the knowledge about LDL-TG in CAD risk prediction among DM and Pre-DM patients was very limited. In the present study, we investigated the prognosis of LDL-TG in real-world patients with different glucose metabolism status and stressed the predictive value of LDL-TG in DM and Pre-DM. In total population, 


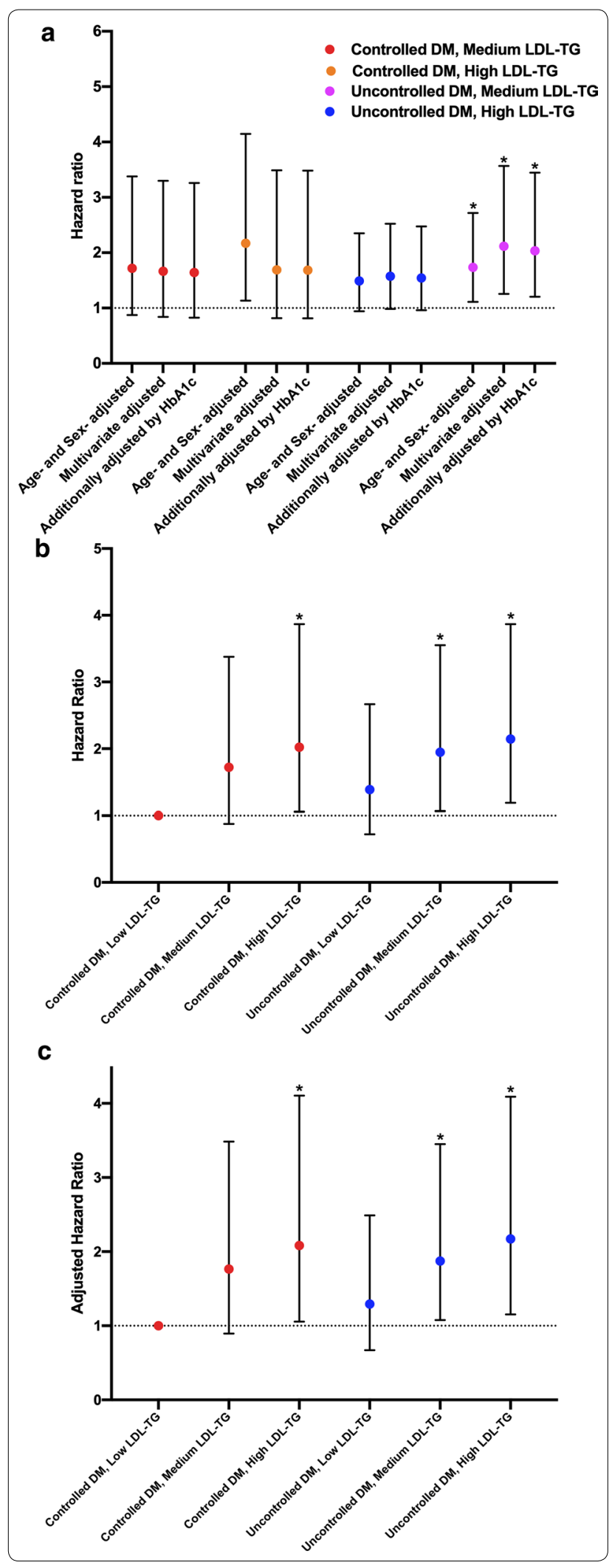

Fig. 4 a Adjusted HR for medium and high LDL-TG in controlled and uncontrolled DM (b) unadjusted and (c) adjusted HR according to both LDL-TG (low T1, medium T2 and high T3) and DM controlled status (well controlled DM plus low LDL-TG as reference). Multivariate adjusted model included age, sex, body mass index, smoking, hypertension, family history of coronary artery disease, Gensini score, left ventricular ejection fraction, LDL-cholesterol, HDL-cholesterol, triglycerides, baseline statin use, and antidiabetic drugs. ${ }^{*}$ for $p<0.05$

DM but not Pre-DM patients presented higher risk of MACEs, which further validated the findings of previous studies $[7,26]$. Notably, when LDL-TG level was incorporated as a stratifying factor in patients with different glucose metabolism status, the risk for those with high LDL-TG plus Pre-DM and high LDL-TG plus DM reached approximately 2-fold. Similarly, within DM group, patients with uncontrolled DM and high LDL-TG were least likely to be free of events, indicating an association of lack of glucose control with dyslipidemia. Our results may be instrumental in risk assessment for CAD patients with impaired glucose metabolism undergoing statin therapy, the so-called residual risk [35].

The results of this study have some practical implications. Firstly, measurement of LDL-TG may bring cardiovascular beneficial effect in clinical practice for its independent association with MACEs. Secondly, the results indicated that LDL-TG levels might be the most powerful marker among all the TGs in lipoprotein and a potential treatment target in DM. As previously reported, TNT trial, lipid lowering with statins reduced TGs in lipoprotein and resulted in a significant lower risk of MACEs [36]. Monoclonal antibodies to proprotein convertase subtilisin/kexin type 9 (PCSK9), alirocumab, could also significantly reduce plasma TG-rich lipoproteins in DM subjects [37]. More studies regarding the potential ways of LDL-TG reduction is in need. Thirdly, we once again concluded that the association between Pre-DM and MACEs might be attributable to the coexistence of other risk factors but our results also indicated that risk assessment by traditional lipid parameters may not be fully adequate [14].

This study has several limitations. First it only included Chinese patients with stable CAD, therefore, data from other ethnicity individuals is necessary; Second, the level of LDL-TG was only measured once in the fasting state and we cannot be sure about fluctuations of biomarkers during follow-up, even so the results were still robust; Third, although the incremental effect of adding LDL-TG into risk model was minor, it was consistent with previous findings. Finally, not all parameters reflecting glucose metabolism were measured due to the clinical and realworld nature of this study population. 


\section{Conclusions}

In conclusion, the current data showed, for the first time, that patients with high LDL-TG and DM or Pre-DM had significantly higher risk of MACEs, a finding that was in accord with robust epidemiological and genetic evidence of the active roles of TG-enriched lipoproteins in atherogenesis. It also suggested that incorporating LDL-TG for cardiovascular risk stratification might be beneficial in identifying higher risk patients with stable CAD and PreDM or DM.

\section{Supplementary information}

Supplementary information accompanies this paper at https://doi. org/10.1186/s12933-020-01125-1.

Additional file 1. Additional figures and tables.

\section{Abbreviations}

NGR: Normal glucose regulation; Pre-DM: Pre-diabetes; DM: Diabetes mellitus; CAD: Coronary artery disease; MACEs: Major adverse cardiovascular events; PCI: Percutaneous coronary artery intervention; CABG: Coronary artery bypass grafting; BMI: Body mass index; HbA1c: haemoglobin A1 c; TC: Total cholesterol; TG: Triglyceride; LDL-C: Low density lipoprotein cholesterol; HDL-C: High density lipoprotein cholesterol; LDL-TG: Low density lipoprotein triglyceride; GS: Gensini score.

\section{Acknowledgements}

The authors thank all the staff and participants of this study for their important contributions.

\section{Authors' contributions}

$\mathrm{J}-\mathrm{L}$ and $\mathrm{H}-\mathrm{WZ}$ completed the project, analyzed the data, and wrote the manuscript. QH and Y-FL contributed to the collections of data. $Y-X C, H-H L$ and $Y Z$, contributed to data collection. Y-LG, N-QW and C-GZ contributed to recruitment of patients and clinical diagnosis of disease and data collection. YG, C-JC, $G L, J S$ and QD contributed to the collections of clinical data and procedure of laboratory examination. RDS has contributed by critically revising the manuscript. J-JL designed the study, interpreted data, and contributed to critically revising the manuscript. All authors have approved the final article. J-JL is the guarantor of this work and, as such, had full access to all the data in the study and takes responsibility for the integrity of the data and the accuracy of the data analysis. All authors read and approved the final manuscript.

\section{Funding}

This work was partially supported by the Capital Health Development Fund (201614035) and CAMS Major Collaborative Innovation Project (2016-I2M -1-011) awarded to Dr. Jian-Jun Li, MD, PhD. Raul D. Santos is a recipient of a scholarship from Conselho Nacional de Pesquisa e Desenvolvimento Tecnológico (CNPq), Brazil \#303734/2018-3. The study sponsors did not participate in the study design; the collection, analysis, or interpretation of data; the writing of the report; or the decision to submit the paper for publication.

\section{Availability of data and materials}

The datasets used and/or analyzed during the current study are not publicly available but are available from the corresponding author on reasonable request.

\section{Ethics approval and consent to participate}

Our study complied with the Declaration of Helsinki and was approved by the hospital's ethical review board (Fu Wai Hospital \& National Center for Cardiovascular Diseases, Beijing, China). Informed written consents were obtained from all patients enrolled in this study.

\section{Consent for publication}

Not applicable.

\section{Competing interests}

Raul D. Santos has received honoraria related to consulting, research and/or speaker activities from: Ache, Amgen, Astra Zeneca, Esperion, Kowa, NovoNordisk, Merck, MSD, Pfizer, PTC and Sanofi/Regeneron. All other authors declare no conflicts of interest.

\section{Author details}

1 State Key Laboratory of Cardiovascular Disease, Fu Wai Hospital, National Center for Cardiovascular Diseases, Chinese Academy of Medical Sciences, Peking Union Medical College, BeiLiShi Road 167, Beijing 100037, China. 2 Department of Cardiology, Xuanwu Hospital, Capital Medical University, Beijing 100053, China. ${ }^{3}$ Department of Cardiology, Beijing Anzhen Hospital, Capital Medical University, Beijing 100029, China. ${ }^{4}$ Hospital Israelita Albert Einstein, Sao Paulo, Brazil. ${ }^{5}$ Heart Institute (InCor), University of Sao Paulo Medical School Hospital, Sao Paulo, Brazil.

Received: 7 June 2020 Accepted: 12 September 2020

Published online: 27 September 2020

\section{References}

1. Sarwar N, Danesh J, Eiriksdottir G, Sigurdsson G, Wareham N, Bingham S, Boekholdt SM, Khaw KT, Gudnason V. Triglycerides and the risk of coronary heart disease: 10,158 incident cases among 262,525 participants in 29 Western prospective studies. Circulation. 2007;115(4):450-8.

2. Reiner Z. Hypertriglyceridaemia and risk of coronary artery disease. Nat Rev Cardiol. 2017:14(7):401-11.

3. Miller M, Cannon CP, Murphy SA, Qin J, Ray KK, Braunwald E. Investigators PI-T: impact of triglyceride levels beyond low-density lipoprotein cholesterol after acute coronary syndrome in the PROVE IT-TIMI 22 trial. J Am Coll Cardiol. 2008:51(7):724-30.

4. Elam MB, Ginsberg HN, Lovato LC, Corson M, Largay J, Leiter LA, Lopez C, O'Connor PJ, Sweeney ME, Weiss D, Friedewald WT, Buse JB, Gerstein HC, Probstfield J, Grimm R, Ismail-Beigi F, Goff DC Jr, Fleg JL, Rosenberg $Y$, Byington RP. Association of fenofibrate therapy with long-term cardiovascular risk in statin-treated patients with type 2 diabetes. JAMA Cardiol. 2017;2(4):370-80.

5. Investigators OT, Bosch J, Gerstein HC, Dagenais GR, Diaz R, Dyal L, Jung $H$, Maggiono AP, Probstfield J, Ramachandran A, Riddle MC, Ryden LE, Yusuf S. $n-3$ fatty acids and cardiovascular outcomes in patients with dysglycemia. N Engl J Med. 2012;367(4):309-18.

6. Bhatt DL, Steg PG, Miller M, Brinton EA, Jacobson TA, Ketchum SB, Doyle RT Jr, Juliano RA, Jiao L, Granowitz C, Tardif JC, Ballantyne CM, Investigators R-I. Cardiovascular risk reduction with icosapent ethyl for hypertriglyceridemia. N Engl J Med. 2019;380(1):11-22.

7. Saeed A, Feofanova EV, Yu B, Sun W, Virani SS, Nambi V, Coresh J, Guild CS, Boerwinkle E, Ballantyne CM, Hoogeveen RC. Remnant-like particle cholesterol, low-density lipoprotein triglycerides, and incident cardiovascular disease. J Am Coll Cardiol. 2018:72(2):156-69.

8. Varbo A, Benn M, Tybjaerg-Hansen A, Jorgensen AB, Frikke-Schmidt R, Nordestgaard BG. Remnant cholesterol as a causal risk factor for ischemic heart disease. J Am Coll Cardiol. 2013;61(4):427-36.

9. Ding $X H$, Ye $P$, Wang $X N$, Cao RH, Yang $X$, Xiao WK, Zhang $Y$, Bai $Y Y$, Wu HM. The predictive value of baseline LDL-TG level on major adverse cardiovascular events in a followed up cohort population. Eur Rev Med Pharmacol Sci. 2017;21(5):1060-4.

10. McKeone BJ, Patsch JR, Pownall HJ. Plasma triglycerides determine low density lipoprotein composition, physical properties, and cell-specific binding in cultured cells. J Clin Invest. 1993;91(5):1926-33.

11. Miller M. Low-density lipoprotein triglycerides: widening the atherogenic landscape in CVD risk assessment. J Am Coll Cardiol. 2018;72(2):170-2.

12. Marz W, Scharnagl H, Winkler K, Tiran A, Nauck M, Boehm BO, Winkelmann BR. Low-density lipoprotein triglycerides associated with low-grade systemic inflammation, adhesion molecules, and angiographic coronary artery disease: the Ludwigshafen Risk and Cardiovascular Health study. Circulation. 2004;110(19):3068-74.

13. Cosentino F, Grant PJ, Aboyans V, Bailey CJ, Ceriello A, Delgado V, Federici M, Filippatos G, Grobbee DE, Hansen TB, Huikuri HV, Johansson I, Juni P, Lettino M, Marx N, Mellbin LG, Ostgren CJ, Rocca B, Roffi M, Sattar N, 
Seferovic PM, Sousa-Uva M, Valensi P, Wheeler DC. 2019 ESC Guidelines on diabetes, pre-diabetes, and cardiovascular diseases developed in collaboration with the EASD. Eur Heart J. 2020:41(2):255-323.

14. Jin JL, Cao YX, Zhang HW, Sun D, Hua Q, Li YF, Guo YL, Wu NQ, Zhu CG, Gao Y, Dong QT, Liu HH, Dong Q, Li JJ. Lipoprotein(a) and cardiovascular outcomes in patients with coronary artery disease and prediabetes or diabetes. Diabetes Care. 2019;42(7):1312-8.

15. Liu HH, Cao YX, Li S, Guo YL, Zhu CG, Wu NQ, Gao Y, Dong QT, Zhao $X$, Zhang Y, Sun D, Li JJ. Impacts of prediabetes mellitus alone or plus hypertension on the coronary severity and cardiovascular outcomes. Hypertension. 2018;71(6):1039-46.

16. Cho NH, Shaw JE, Karuranga S, Huang Y, da Rocha Fernandes JD, Ohlrogge AW, Malanda B. IDF Diabetes Atlas: global estimates of diabetes prevalence for 2017 and projections for 2045. Diabetes Res Clin Pract. 2018;138:271-81.

17. Coopmans C, Zhou TL, Henry RMA, Heijman J, Schaper NC, Koster A, Schram MT, Kallen C, Wesselius A, den Engelsman RJA, Crijns H, Stehouwer CDA. Both prediabetes and type 2 diabetes are associated with lower heart rate variability: The Maastricht Study. Diabetes Care. 2020:43:1126-33.

18. Lee JS, Chang PY, Zhang Y, Kizer JR, Best LG, Howard BV. Triglyceride and HDL-C dyslipidemia and risks of coronary heart disease and ischemic stroke by glycemic dysregulation status: The Strong Heart Study. Diabetes Care. 2017:40(4):529-37.

19. American Diabetes Association. Classification and diagnosis of diabetes: standards of medical care in diabetes-2019. Diabetes Care. 2019:42(1):S13-28.

20. Ito Y, Ohta M, Ikezaki H, Hirao Y, Machida A, Schaefer EJ, Furusyo N. Development and population results of a fully automated homogeneous assay for LDL triglyceride. J Appl Lab Med. 2018;2(5):746-56.

21. Gensini GG. A more meaningful scoring system for determining the severity of coronary heart disease. Am J Cardiol. 1983;51(3):606.

22. Shemesh E, Zafrir B. Hypertriglyceridemia-related pancreatitis in patients with type 2 diabetes: links and risks. Diabetes Metab Syndr Obes. 2019;12:2041-52.

23. Song PK, Man QQ, Li H, Pang SJ, Jia SS, Li YQ, He L, Zhao WH, Zhang J. Trends in lipids level and dyslipidemia among Chinese adults, 2002-2015. Biomed Environ Sci. 2019;32(8):559-70.

24. Ference BA, Kastelein JJP, Ray KK, Ginsberg HN, Chapman MJ, Packard CJ, Laufs U, Oliver-Williams C, Wood AM, Butterworth AS, Di Angelantonio E, Danesh J, Nicholls SJ, Bhatt DL, Sabatine MS, Catapano AL. Association of triglyceride-lowering LPL variants and LDL-C-lowering LDLR variants with risk of coronary heart disease. JAMA. 2019;321(4):364-73.

25. Marston NA, Giugliano RP, Im K, Silverman MG, O'Donoghue ML, Wiviott $S D$, Ference BA, Sabatine MS. Association between triglyceride lowering and reduction of cardiovascular risk across multiple lipid-lowering therapeutic classes: a systematic review and meta-regression analysis of randomized controlled trials. Circulation. 2019;140(16):1308-17.

26. Silbernagel G, Scharnagl H, Kleber ME, Delgado G, Stojakovic T, Laaksonen R, Erdmann J, Rankinen T, Bouchard C, Landmesser U, Schunkert H, Marz W, Grammer TB. LDL triglycerides, hepatic lipase activity, and coronary artery disease: an epidemiologic and Mendelian randomization study. Atherosclerosis. 2019;282:37-44

27. Albers JJ, Slee A, Fleg JL, O'Brien KD, Marcovina SM. Relationship of baseline HDL subclasses, small dense LDL and LDL triglyceride to cardiovascular events in the AIM-HIGH clinical trial. Atherosclerosis. 2016;251:454-9.

28. Guyton JR, Slee AE, Anderson T, Fleg JL, Goldberg RB, Kashyap ML, Marcovina SM, Nash SD, O'Brien KD, Weintraub WS, Xu P, Zhao XQ, Boden WE. Relationship of lipoproteins to cardiovascular events: the AIM-HIGH Trial (Atherothrombosis Intervention in Metabolic Syndrome With Low HDL/
High Triglycerides and Impact on Global Health Outcomes). J Am Coll Cardiol. 2013;62(17):1580-4.

29. Giugliano RP, Cannon CP, Blazing MA, Nicolau JC, Corbalán R, Špinar J, Park JG, White JA, Bohula EA, Braunwald E. Benefit of adding ezetimibe to statin therapy on cardiovascular outcomes and safety in patients with versus without diabetes mellitus: results from IMPROVE-IT (Improved Reduction of Outcomes: Vytorin Efficacy International Trial). Circulation. 2018;137(15):1571-82.

30. Sabatine MS, Leiter LA, Wiviott SD, Giugliano RP, Deedwania P, De Ferrari GM, Murphy SA, Kuder JF, Gouni-Berthold I, Lewis BS, Handelsman Y, Pineda AL, Honarpour N, Keech AC, Sever PS, Pedersen TR. Cardiovascular safety and efficacy of the PCSK9 inhibitor evolocumab in patients with and without diabetes and the effect of evolocumab on glycaemia and risk of new-onset diabetes: a prespecified analysis of the FOURIER randomised controlled trial. Lancet Diabetes Endocrinol. 2017;5(12):941-50.

31. Ray KK, Colhoun HM, Szarek M, Baccara-Dinet M, Bhatt DL, Bittner VA, Budaj AJ, Diaz R, Goodman SG, Hanotin C, Harrington RA, Jukema JW, Loizeau V, Lopes RD, Moryusef A, Murin J, Pordy R, Ristic AD, Roe MT, Tuñón J, White HD, Zeiher AM, Schwartz GG, Steg PG. Effects of alirocumab on cardiovascular and metabolic outcomes after acute coronary syndrome in patients with or without diabetes: a prespecified analysis of the ODYSSEY OUTCOMES randomised controlled trial. Lancet Diabetes Endocrinol. 2019;7(8):618-28.

32. Ye X, Kong W, Zafar MI, Chen LL. Serum triglycerides as a risk factor for cardiovascular diseases in type 2 diabetes mellitus: a systematic review and meta-analysis of prospective studies. Cardiovasc Diabetol. 2019;18(1):48.

33. Hsu H, Hsu P, Cheng MH, Ito Y, Kanda E, Schaefer EJ, Ai M. Lipoprotein Subfractions and Glucose Homeostasis in Prediabetes and Diabetes in Taiwan. J Atheroscler Thromb. 2019;26(10):890-914.

34. Ference BA, Ginsberg HN, Graham I, Ray KK, Packard CJ, Bruckert E, Hegele RA, Krauss RM, Raal FJ, Schunkert H, Watts GF, Borén J, Fazio S, Horton JD, Masana L, Nicholls SJ, Nordestgaard BG, van de Sluis B, Taskinen MR, Tokgözoglu L, Landmesser U, Laufs U, Wiklund O, Stock JK, Chapman MJ, Catapano AL. Low-density lipoproteins cause atherosclerotic cardiovascular disease. 1. Evidence from genetic, epidemiologic, and clinical studies. A consensus statement from the European Atherosclerosis Society Consensus Panel. Eur Heart J. 2017;38(32):2459-72.

35. Fruchart JC, Santos RD, Yamashita S, Libby P. Residual vascular risk in diabetes - Will the SPPARM alpha concept hold the key? Diabetes Metab Syndr. 2019;13(4):2723-5.

36. Vallejo-Vaz AJ, Fayyad R, Boekholdt SM, Hovingh GK, Kastelein JJ, Melamed S, Barter P, Waters DD, Ray KK. Triglyceride-rich lipoprotein cholesterol and risk of cardiovascular events among patients receiving statin therapy in the TNT trial. Circulation. 2018;138(8):770-81.

37. Colhoun HM, Leiter LA, Müller-Wieland D, Cariou B, Ray KK, Tinahones FJ, Domenger C, Letierce A, Israel M, Samuel R, Del Prato S. Effect of alirocumab on individuals with type 2 diabetes, high triglycerides, and low high-density lipoprotein cholesterol. Cardiovasc Diabetol. 2020;19(1):14.

\section{Publisher's Note}

Springer Nature remains neutral with regard to jurisdictional claims in published maps and institutional affiliations.

Ready to submit your research? Choose BMC and benefit from:

- fast, convenient online submission

- thorough peer review by experienced researchers in your field

- rapid publication on acceptance

- support for research data, including large and complex data types

- gold Open Access which fosters wider collaboration and increased citations

- maximum visibility for your research: over 100M website views per year

At BMC, research is always in progress.

Learn more biomedcentral.com/submissions 